\title{
Algebraic and Analytic Properties of the One-Dimensional Hubbard Model
}

\author{
Frank Göhmann洶 and Shuichi Murakami垖 \\ ${ }^{\dagger}$ Department of Physics, Faculty of Science, \\ University of Tokyo, \\ Hongo 7-3-1, Bunkyo-ku, Tokyo 113, Japan \\ ${ }^{\ddagger}$ Department of Applied Physics, Faculty of Engineering, \\ University of Tokyo, \\ Hongo 7-3-1, Bunkyo-ku, Tokyo 113, Japan
}

\begin{abstract}
We reconsider the quantum inverse scattering approach to the one-dimensional Hubbard model and work out some of its basic features so far omitted in the literature. It is our aim to show that $R$-matrix and monodromy matrix of the Hubbard model, which are known since ten years now, have good elementary properties. We provide a meromorphic parametrization of the transfer matrix in terms of elliptic functions. We identify the momentum operator for lattice fermions in the expansion of the transfer matrix with respect to the spectral parameter and thereby show the locality and translational invariance of all higher conserved quantities. We work out the transformation properties of the monodromy matrix under the $\mathrm{su}(2)$ Lie algebra of rotations and under the $\eta$-pairing su(2) Lie algebra. Our results imply su(2) $\oplus \operatorname{su}(2)$ invariance of the transfer matrix for the model on a chain with an even number of sites.
\end{abstract}

*e-mail: frank@monet.phys.s.u-tokyo.ac.jp

§e-mail: murakami@appi.t.u-tokyo.ac.jp

`Address from Oct. 1996: Physikalisches Institut der Universität Bayreuth, TP1, 95440 Bayreuth, Germany 


\section{Introduction}

The one-dimensional Hubbard model is one of the most thoroughly studied integrable quantum systems with applications in solid state physics. Starting with the seminal article [1] of Lieb and $\mathrm{Wu}$ lots of its physical properties have been worked out exactly [2]. For the case of half-filled band, in particular, a complete picture of its elementary excitations is available by now [3, 4. All excited states are scattering states of only four quasiparticles, two of which carry spin but no charge, whereas the other two carry charge but no spin. The $S$-matrix of these quasiparticles has been calculated exactly. These achievements give a precise meaning to the notion of spin-charge separation in one-dimensional solids.

All exact results on physical properties of the one-dimensional Hubbard model obtained so far rely on the extensive use of the coordinate Bethe Ansatz. Since Bethe wave functions, however, are difficult to handle, any exact calculation of local quantities going beyond the long distance asymptotics of correlation functions [5] seems to demand for an algebraic treatment. Moreover, an algebraic treatment is likely to facilitate the calculation of the thermodynamical properties of the model [6].

At present we know two algebraic structures related to the Hubbard model, a graded Yang-Baxter algebra, developed in the works of Shastry [7, 8, 9] and Olmedilla et al. 110, 11, 12 and a representation of the $\mathrm{Y}(\mathrm{su}(2))$ Yangian quantum group commuting with the Hubbard Hamiltonian, which was discovered by Uglov and Korepin [13]. The relation of these two notions was recently exposed by the authors 14.

Although $R$-matrix and $L$-matrix of the Hubbard model are known since long, it took nearly ten years before it was shown that the $R$-matrix satisfies the Yang-Baxter equation [15]. An algebraic Bethe Ansatz was performed only recently in a remarkable preprint by Ramos and Martins [16]. Every progress in the development of an algebraic approach was hindered before by the complexity of $R$-matrix and monodromy matrix and by several unusual features of these basic tools of the quantum inverse scattering method. The monodromy matrix is $4 \times 4$ rather than $3 \times 3$, as one might have guessed naively from the fact that there are two levels of Bethe Ansatz equations. It further 
seems to be impossible to find a parametrization of the $R$-matrix, such that it becomes a function of the difference of the spectral parameters.

Amazingly, not even the most elementary properties of $R$-matrix and monodromy matrix have been worked out so far. For that reason we look again at the construction of the graded Yang-Baxter algebra. We begin with a description of the spin model [7, 8, 9] that is related to the Hubbard model by means of a Jordan-Wigner transformation in section 2. Our account is based on the Yang-Baxter equation. In section 3 we show that there exists a meromorphic parametrization of the transfer matrix in terms of elliptic functions. Section 4 is devoted to the rederivation of the graded Yang-Baxter algebra [11. We show how to treat general twisted boundary conditions. As a byproduct we find a simple method to obtain higher conserved quantities of the Hubbard model from their counterparts for the spin model. These conserved quantities are generated by the graded trace of the fermionic monodromy matrix. We identify the momentum operator for fermions in the zeroth order of the expansion of this generating function with respect to the spectral parameter. Thus all higher conserved quantities are local and translational invariant. In section 5 we derive the properties of the monodromy matrix under a combined particle-hole and gauge transformation, which is characteristical for the Hubbard Hamiltonian. It turns out that the graded trace of the monodromy matrix is invariant under this transformation, if the model is considered on a chain with an even number of sites. In section 6 we investigate the behaviour of the monodromy matrix under $\mathrm{su}(2)$ transformations. Our results are complementary to the work of Ramos and Martins [16] and should provide a means to discuss the symmetry properties of quasiparticles within the algebraic approach. Like the Hamiltonian, the graded trace of the monodromy matrix turns out to be invariant under rotations of the spins and, if we consider an even number of sites, also under the $\eta$-pairing $\mathrm{su}(2)$ Lie algebra 17 , [18, 19, 20]. In order to make our representation self-contained, we expose the $R$-matrix along with a list of relations among its elements in appendix A. Appendix B provides a detailed discussion of the momentum operator on a lattice. In appendix $\mathrm{C}$ it is shown how to obtain the momentum operator from a monodromy matrix for free fermions. 


\section{The spin model}

As all one-dimensional fermionic models the Hubbard model is related to a certain spin chain by a Jordan-Wigner transformation. The Yang-Baxter algebra corresponding to this spin chain is easier to formulate and closer to intuition than the graded Yang-Baxter algebra of the Hubbard model. In fact, Shastry in his seminal articles [7, 8, 9] was using the language of spins rather than the language of electrons. The graded form of the Yang-Baxter algebra having the advantage of being defined directly in terms of fermi operators was derived later by Olmedilla et al. [10, 11, 12]. Let us follow the historical route here and start with a description of the spin model. Its Hamiltonian is

$$
H=\sum_{j=1}^{L}\left(\sigma_{j \tau}^{+} \sigma_{j+1 \tau}^{-}+\sigma_{j \tau}^{-} \sigma_{j+1 \tau}^{+}+\frac{U}{4} \sigma_{j \uparrow}^{z} \sigma_{j \downarrow}^{z}\right)
$$

Here and in the following we are using implicit summation over doubly occuring indices. $H$ describes a periodic spin chain of $L$ sites $\left(\sigma_{L+1 \tau}^{ \pm}:=\sigma_{1 \tau}^{ \pm}\right)$with two species of spins, labeled $\uparrow$ and $\downarrow$, at each site. $U$ is the strength of an on-site Ising coupling between the species. The interaction between nearest neighbours is of XX-type for both species independently. Thus, in the limit $U \rightarrow 0$ the model decouples into a pair of noninteracting XX-chains.

As was shown by Shastry, $H$ is the logarithmic derivative of a certain transfer matrix, which can be obtained by appropriately coupling together two copies of the Yang-Baxter algebra of the XX-chain. The $R$-matrix of the XX-chain is

$$
r=\frac{1}{2}\left(a+b+(a-b) \sigma^{z} \otimes \sigma^{z}+\sigma^{x} \otimes \sigma^{x}+\sigma^{y} \otimes \sigma^{y}\right),
$$

where $a$ and $b$ have to satisfy the free fermion condition

$$
a^{2}+b^{2}=1
$$

If we introduce the parametrization $a=\cos (\lambda), b=\sin (\lambda)$, then $r=r(\lambda)$ satisfies the Yang-Baxter equation in the form

$$
r_{12}(\lambda-\mu) r_{13}(\lambda) r_{23}(\mu)=r_{23}(\mu) r_{13}(\lambda) r_{12}(\lambda-\mu)
$$


which is sometimes called difference form of the Yang-Baxter equation. $r(\lambda)$ is regular, i.e. $r(0)=P$, the permutation of the two factors of $\mathbf{C}^{2} \otimes \mathbf{C}^{2}$. As usual, the indices in (四) refer to the canonical embeddings of $r(\lambda)$ into $\mathbf{C}^{2} \otimes \mathbf{C}^{2} \otimes \mathbf{C}^{2}$. The $L$-matrix $l(\lambda)$ of the XX-chain is the fundamental representation $l(\lambda)=r(\lambda)$ of the Yang-Baxter algebra,

$$
\check{r}(\lambda-\mu)(l(\lambda) \otimes l(\mu))=(l(\mu) \otimes l(\lambda)) \check{r}(\lambda-\mu) \quad,
$$

generated by $\check{r}(\lambda):=\operatorname{Pr}(\lambda)$. As usual, $l(\lambda)$ in eq. (5) has to be understood as matrix in auxiliary space with entries acting on a quantum space.

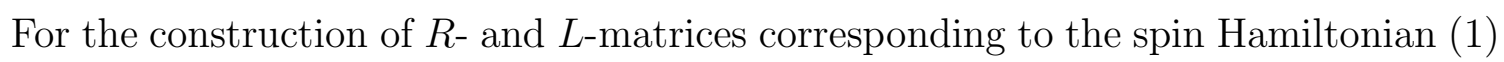
we have to duplicate the above construction by attaching a label referring to the spin species to each $\sigma$-matrix. The matrix $r(\lambda)$ is replaced by $r_{\tau}(\lambda)(\tau=\uparrow, \downarrow)$, and we may redefine $r(\lambda)$ as

$$
r(\lambda):=r_{\uparrow}(\lambda) r_{\downarrow}(\lambda)
$$

This new $R$-matrix obviously satisfies (ब4) and is again regular in the sense that $r(0)=$ $P_{\uparrow} P_{\downarrow}=: P$ is a permutation operator. Whenever an explicit matrix representation is required we will use the convention $\sigma_{\uparrow}^{\alpha}=\sigma^{\alpha} \otimes I_{2}, \sigma_{\downarrow}^{\alpha}=I_{2} \otimes \sigma^{\alpha}$, where $I_{2}$ denotes the $2 \times 2$ unit matrix. Correspondingly, the $4 \times 4$ unit matrix, which will be needed below, is denoted by $I_{4}$. Now Shastry's $R$-matrix associated to the Hamiltonian (11) reads

$$
R(\lambda, \mu \mid h, l):=\operatorname{ch}(h-l) \frac{r(\lambda-\mu)}{\cos (\lambda-\mu)}+\operatorname{sh}(h-l) \frac{r(\lambda+\mu)}{\cos (\lambda+\mu)} \sigma^{z} \otimes \sigma^{z} \otimes I_{4} \quad .
$$

This is a four parameter family of $16 \times 16$-matrices. It was shown by Shiroishi and Wadati [15], that it satisfies the Yang-Baxter equation in the form

$$
R_{12}(\lambda, \mu \mid h, l) R_{13}(\lambda, \nu \mid h, m) R_{23}(\mu, \nu \mid l, m)=R_{23}(\mu, \nu \mid l, m) R_{13}(\lambda, \nu \mid h, m) R_{12}(\lambda, \mu \mid h, l)
$$

provided that the parameters are constrained by the equations

$$
\frac{U}{4}=\frac{\operatorname{sh}(2 h)}{\sin (2 \lambda)}=\frac{\operatorname{sh}(2 l)}{\sin (2 \mu)}=\frac{\operatorname{sh}(2 m)}{\sin (2 \nu)} .
$$

This constraint can be satisfied for arbitrarily small $m$ and $\nu$. Hence,

$$
L_{j k}(\lambda \mid h):=\cos (\lambda) R_{j k}(\lambda, 0 \mid h, 0)=r_{j k}(\lambda) e^{h \sigma_{j \uparrow}^{z} \sigma_{j \downarrow}^{z}}
$$


is a representation of the Yang-Baxter algebra generated by the $R$-matrix (77). The index $j$ in (10) refers to the auxiliary space, the index $k$ to the quantum space. Note that $L_{j k}(\lambda \mid h) \neq L_{k j}(\lambda \mid h) . \quad L_{j k}(\lambda \mid h)$ is a tensor product of two XX-chain $L$-matrices coupled in auxiliary space. If we solve the first equation in (9) for $h$ and choose the branch of solution properly, then $h(\lambda=0)=0$, and $L_{j k}$ according to (10) is again regular. For $U=0$ the constraint (9) is satisfied by $h=0$ for all $\lambda$. We get back the free model as it should be by construction, and the $R$-matrix (7) becomes a solution of the Yang-Baxter equation in difference form (4). On the other extreme, we can carry out the limit $U \rightarrow \infty$ after properly rescaling $\lambda \rightarrow \lambda / U$. Then the constraint (9) becomes $\lambda=2 \operatorname{sh}(2 h)$. However, $\lambda$ disappears from the definitions of $L_{j k}$ and $R_{j k}$, since $\cos (\lambda / U) \rightarrow 1$ and $\sin (\lambda / U) \rightarrow 0$ for every $\lambda$. Thus $r_{j k}((\lambda-\mu) / U) \rightarrow P_{j k}$, and

$$
R_{j k}(\lambda, \mu \mid h, l) \rightarrow L_{j k}(0 \mid h-l)=P_{j k} e^{(h-l) \sigma_{j \uparrow}^{z} \sigma_{j \downarrow}^{z}} .
$$

It is easily verified that the expression on the right hand side of (11) satisfies the YangBaxter equation in difference form (4) and that the corresponding Hamiltonian is the on-site part of (11) with $U=1$. Hence $\lambda$ is the natural spectral parameter of the model at zero coupling, whereas $h$ is the natural spectral parameter in the strong coupling limit. The algebra (7), (8), (9) interpolates between these limits. For the remainder of this article we will suppress the arguments $h$ and $l$ of the $R$ - and $L$-matrices, assuming that they are given as functions of $\lambda$ and $\mu$ by the constraint (9).

To finish the description of the spin model let us introduce its monodromy matrix,

$$
T_{L}(\lambda):=L_{a L}(\lambda) \ldots L_{a 1}(\lambda)
$$

The index $a$ in this definition refers to the auxiliary space. The transfer matrix of the spin model, $t(\lambda)$, is the trace of $T_{L}(\lambda)$ over the auxiliary space. It follows from the regularity of $L_{j k}$ that $\tilde{U}:=t(0)$ is the shift operator for spins,

$$
\tilde{U} \sigma_{j \tau}^{\alpha}=\sigma_{j+1 \tau}^{\alpha} \tilde{U} \quad, \quad j=1, \ldots, L \quad, \quad \tau=\uparrow, \downarrow \quad .
$$

A brief calculation yields the derivative of the $L$-matrix at zero spectral parameter,

$$
\dot{L}_{j k}(0) P_{j k}=\sigma_{j \tau}^{+} \sigma_{k \tau}^{-}+\sigma_{j \tau}^{-} \sigma_{k \tau}^{+}+\frac{U}{4} \sigma_{k \uparrow}^{z} \sigma_{k \downarrow}^{z} .
$$


This equation implies that the Hamiltonian (1) is obtained from the transfer matrix $t(\lambda)$ as logarithmic derivative, $H=\left.d_{\lambda} \ln (t(\lambda))\right|_{\lambda=0}$.

\section{A meromorphic parametrization of the transfer matrix}

The considerations in this section were motivated by two facts. First, in case of the eight vertex model Baxter's meromorphic parametrization of the $R$-matrix yields a solution of difference form of the Yang-Baxter equation [21]. Second, the transfer matrix enters certain functional equations, the solutions of which usually require strong analytic properties.

Let us solve the constraint (9) for $e^{2 h}$,

$$
e^{2 h}=\frac{U \sin (2 \lambda)}{4}+\sqrt{1+\frac{U^{2} \sin ^{2}(2 \lambda)}{16}} .
$$

The only possibility to remove the square root on the right hand side is by replacing $2 \lambda$ by $\operatorname{am}(u)$, the amplitude function and setting $k=\mathrm{i} U / 4$. Then $e^{2 h}$ is expressed in terms of Jacobi elliptic functions as

$$
e^{2 h}=\operatorname{dn}(u)-\mathrm{i} k \operatorname{sn}(u) .
$$

Because of the homogeneity of the Yang-Baxter algebra, we may multiply $L_{j k}(u)$ by $e^{h}$. Then $h(u)$ enters into the definition of the monodromy matrix $T_{a}(u)$ only as meromorphic function $e^{2 h}$ of the redefined spectral parameter $u$. Letting $A:=(a+b) / 2$ and $B=$ $(a-b) / 2$ we see that the matrix $r_{j k}$ in the definition of the $L$-matrix is of the form

$$
r_{j k}=\left(\begin{array}{cccc}
e & o & o & e \\
o & e & e & o \\
o & e & e & o \\
e & o & o & e
\end{array}\right),
$$

where $e$ denotes an even polynomial in $A, B$ and $o$ denotes an odd one. $e$ and $o$ are operators on quantum space $k$, whose precise form is irrelevant for the following 
arguments. The rules $e^{2}=o^{2}=e, e o=o e=o, e+e=e$ and $o+o=o$ imply

$$
\left(\begin{array}{llll}
e & o & o & e \\
o & e & e & o \\
o & e & e & o \\
e & o & o & e
\end{array}\right)^{2}=\left(\begin{array}{llll}
e & o & o & e \\
o & e & e & o \\
o & e & e & o \\
e & o & o & e
\end{array}\right) .
$$

This means that the monodromy matrix (12) is again of the form (17). Therefore $t(u)$ is an even polynomial in $A$ and $B$. In other words, $t(u)$ is a polynomial in $A^{2}, B^{2}$ and $A B$. Since

$$
A^{2}=(1+\operatorname{sn}(u)) / 4 \quad, \quad B^{2}=(1-\operatorname{sn}(u)) / 4 \quad, \quad A B=\operatorname{cn}(u) / 4,
$$

$t(u)$ is a meromorphic function of $u$.

To state the problem of the analytic structure of the $R$-matrix let us go one step back and write again $a$ for $\cos (\lambda)$ and $b$ for $\sin (\lambda)$. Let furthermore $c:=e^{2 h}$. Then $a$, $b$ and $c$ are connected by the free fermion condition (3) and the constraint (9), i.e. $a, b$ and $c$ are lying on a complex curve given by the algebraic equations

$$
a^{2}+b^{2}=1 \quad, \quad c-1 / c=U a b .
$$

This curve may be called the spectral curve of the Hubbard model. Unfortunately we could neither find a meromorphic parametrization of this seemingly simple structure nor assign a geometrical meaning to it.

\section{The Hubbard model}

Applying a Jordan-Wigner transformation to $R$ - and $L$-matrix of the spin model we obtain the graded Yang-Baxter algebra [22] of the Hubbard model [11]. No extra effort is necessary to introduce the grading. It is rather induced by the Jordan-Wigner transformation. Before turning to the fermionic formulation let us perform a gauge transformation of $R$ - and $L$-matrices with $4 \times 4$ transformation matrix

$$
G_{x}:=e^{x\left(\sigma^{z} \otimes \sigma^{z}\right) / 2}
$$


Then

$$
\begin{aligned}
L_{k}(\lambda) & \longrightarrow \tilde{L}_{k}(\lambda):=G_{h} L_{k}(\lambda) G_{h}^{-1}=G_{h}\left(l_{k \uparrow}(\lambda) \otimes l_{k \downarrow}(\lambda)\right) G_{h}, \\
R(\lambda, \mu) & \longrightarrow \tilde{R}(\lambda, \mu):=\left(G_{h} \otimes G_{l}\right) R(\lambda, \mu)\left(G_{h}^{-1} \otimes G_{l}^{-1}\right) .
\end{aligned}
$$

We suppress the auxiliary space index of the $L$-matrix here and in the following and consider $\tilde{L}_{k}(\lambda)$ as $4 \times 4$ matrix with entries acting on quantum space $k$. Unlike $R(\lambda, \mu)$, the transformed matrix $\tilde{R}(\lambda, \mu)$ is symmetric. By use of the definition

$$
\check{R}(\lambda, \mu):=P \tilde{R}(\lambda, \mu)
$$

the Yang-Baxter algebra assumes the form

$$
\check{R}(\lambda, \mu)\left(\tilde{L}_{k}(\lambda) \otimes \tilde{L}_{k}(\mu)\right)=\left(\tilde{L}_{k}(\mu) \otimes \tilde{L}_{k}(\lambda)\right) \check{R}(\lambda, \mu),
$$

which is most convenient for changing to a fermionic formulation [22].

Since the canonical anticommutation relations for fermi operators are invariant under gauge transformations and under particle-hole transformations, there is some freedom in the definition of the Jordan-Wigner transformation. Using the abbreviations

$$
u_{k}:=\sum_{j=1}^{k} n_{j \uparrow} \quad, \quad d_{k}:=\sum_{j=1}^{k} n_{j \downarrow} \quad, k=1, \ldots, L,
$$

where the $n_{j s}$ are electron densities and setting further $u_{0}:=d_{0}:=1$, we take the choice

$$
\begin{gathered}
\sigma_{k \uparrow}^{+}=c_{k \uparrow}^{+} e^{\mathrm{i} \pi u_{k-1}} \quad, \quad \sigma_{k \uparrow}^{-}=c_{k \uparrow} e^{-\mathrm{i} \pi u_{k-1}}, \\
\sigma_{k \downarrow}^{+}=c_{k \downarrow}^{+} e^{\mathrm{i} \pi\left(u_{L}+d_{k-1}\right)} \quad, \quad \sigma_{k \downarrow}^{-}=c_{k \downarrow} e^{-\mathrm{i} \pi\left(u_{L}+d_{k-1}\right)} .
\end{gathered}
$$

The XX $L$-matrices $l_{k \uparrow}(\lambda)$ and $l_{k \downarrow}(\lambda)$ can now be expressed in terms of fermi operators,

$$
\begin{aligned}
& l_{k \uparrow}(\lambda)=e^{-\mathrm{i} \pi u_{k} \sigma^{z} / 2} \mathcal{L}_{k \uparrow}(\lambda) e^{\mathrm{i} \pi u_{k-1} \sigma^{z} / 2}, \\
& l_{k \downarrow}(\lambda)=e^{-\mathrm{i} \pi\left(u_{L}+d_{k}\right) \sigma^{z} / 2} \mathcal{L}_{k \downarrow}(\lambda) e^{\mathrm{i} \pi\left(u_{L}+d_{k-1}\right) \sigma^{z} / 2},
\end{aligned}
$$

where $\mathcal{L}_{k \tau}(\lambda)(\tau=\uparrow, \downarrow)$ is defined as

$$
\mathcal{L}_{k \tau}(\lambda):=\left(\begin{array}{cc}
\sin (\lambda)+\mathrm{i} e^{\mathrm{i} \lambda} n_{k \tau} & c_{k \tau} \\
-\mathrm{i} c_{k \tau}^{+} & \cos (\lambda)-e^{\mathrm{i} \lambda} n_{k \tau}
\end{array}\right) .
$$


$\mathcal{L}_{k \tau}(\lambda)$ is an $L$-matrix for free fermions. Its entries for different quantum space indices $k$ either commute or anticommute. This fact can be formally described by assigning a parity $\pi\left(\mathcal{L}_{k \tau}(\lambda)_{j}^{i}\right)=0,1$ to each matrix element. Call a matrix element even, if its parity is zero, odd if it is one. Odd matrix elements with different quantum space indices anticommute, whereas even elements commute with all elements with different quantum space indices. A grading is a function $p$, which assigns parity to the basis vectors of the auxiliary space. Let $p(i):=p\left(e_{i}\right)$. Then, with the grading $p(1)=0, p(2)=1, p \in Z_{2}$, the elements of the free fermion $L$-matrix have parity $\pi\left(\mathcal{L}_{k \tau}(\lambda)_{j}^{i}\right)=p(i)+p(j)$. The crucial point about these notions is that they allow for the introduction of a graded tensor product $\underset{S}{\otimes}$ which respects comultiplication. Let $\underset{S}{\otimes}$ be defined by the equation

$$
(A \underset{\mathrm{s}}{\otimes} B)_{k l}^{i j}:=(-1)^{(p(i)+p(k)) p(j)} A_{k}^{i} B_{l}^{j} .
$$

Then $(A \underset{\mathrm{S}}{\otimes} B)(C \underset{\mathrm{S}}{\otimes} D)=(A C \underset{\mathrm{S}}{\otimes} B D)$ for all matrices $A, B, C, D$ of the parity defined above. The graded tensor product can be used to formulate a graded Yang-Baxter algebra [22]. Grading and graded tensor product may be defined for arbitrary matrix dimensions.

In appendix $\mathrm{C}$ we give a brief account of the free fermion model which will be needed below to recover the lattice momentum operator from the monodromy matrix of the Hubbard model. The free fermion monodromy matrix is

$$
\mathcal{T}_{L \tau}(\lambda):=\mathcal{L}_{L \tau}(\lambda) \ldots \mathcal{L}_{1 \tau}(\lambda) \quad, \quad(\tau=\uparrow, \downarrow)
$$

Note that our choice of Jordan-Wigner transformation and thus our free fermion $L$ matrix differ from that in [11].

Inserting (29), (30) into (22) we find

$$
\tilde{L}_{k}(\lambda)=W V_{k}^{-1} \mathcal{L}_{k}(\lambda) V_{k-1} W^{-1}
$$

where

$$
\begin{aligned}
V_{k} & :=e^{\mathrm{i} \pi u_{k} \sigma^{z} / 2} \otimes e^{\mathrm{i} \pi\left(u_{L}+d_{k}\right) \sigma^{z} / 2} \\
W & :=\operatorname{diag}(1,1, \mathrm{i}, \mathrm{i}) \\
\mathcal{L}_{k}(\lambda) & =G_{h}\left(\mathcal{L}_{k \uparrow}(\lambda){\underset{\mathrm{S}}{\mathrm{L}}}_{\mathcal{L}_{k \downarrow}}(\lambda)\right) G_{h} .
\end{aligned}
$$


The grading comes in naturally in (37), since the operator $I_{2} \otimes e^{-\mathrm{i} \pi\left(u_{L}+d_{k}\right) \sigma^{z} / 2}$ when moved to the left in the tensor product $l_{k \uparrow}(\lambda) \otimes l_{k \downarrow}(\lambda)$ induces a gauge transformation on $\mathcal{L}_{k \uparrow}(\lambda)$, which affects only the odd elements. We will see below that $\mathcal{L}_{k}(\lambda)$ according to equation (37) is an $L$-matrix for the Hubbard model. The associated $R$-matrix follows from (25). First of all we have

$$
\begin{aligned}
& \tilde{L}_{k}(\lambda) \otimes \tilde{L}_{k}(\mu)= \\
& \quad(W \otimes W)\left(V_{k}^{-1} \otimes V_{k}^{-1}\right) X\left(\mathcal{L}_{k}(\lambda) \underset{\mathrm{S}}{\otimes} \mathcal{L}_{k}(\mu)\right) X^{-1}\left(V_{k-1} \otimes V_{k-1}\right)\left(W^{-1} \otimes W^{-1}\right),
\end{aligned}
$$

where $\underset{S}{\otimes}$ is a graded tensor product of $4 \times 4$ matrices (cf. (32)) with grading $p(1)=$ $p(4)=0, p(2)=p(3)=1$. We are using the same symbol for graded tensor products of $2 \times 2$ and $4 \times 4$ matrices. For $4 \times 4$ matrices the grading will always be as above, and for $2 \times 2$ matrices we will use the grading introduced below (32). The matrix $X$ in (38) is the diagonal matrix

$$
X:=\sigma^{z} \otimes \operatorname{diag}(1, \mathrm{i}, \mathrm{i}, 1) \otimes I_{2}
$$

Let

$$
\Gamma:=\operatorname{diag}\left(e^{\mathrm{i} \alpha}, e^{\mathrm{i} \beta}, e^{\mathrm{i} \gamma}, e^{\mathrm{i} \delta}\right)
$$

where $\alpha, \beta, \gamma, \delta$ may generally be mutually commuting operators. Then

$$
\begin{gathered}
{[\check{R}(\lambda, \mu), \Gamma \otimes \Gamma]=0,} \\
\Leftrightarrow \quad \alpha+\delta=\beta+\gamma \bmod 2 \pi .
\end{gathered}
$$

Since $V_{k}$ and $\mathrm{W}$ are of the form (40) and satisfy (42), we may infer from (38) that

$$
\mathcal{R}(\lambda, \mu)\left(\mathcal{L}_{k}(\lambda) \underset{\mathrm{S}}{\otimes} \mathcal{L}_{k}(\mu)\right)=\left(\mathcal{L}_{k}(\mu) \underset{\mathrm{S}}{\otimes} \mathcal{L}_{k}(\lambda)\right) \mathcal{R}(\lambda, \mu)
$$

where

$$
\mathcal{R}(\lambda, \mu)=X^{-1} \check{R}(\lambda, \mu) X
$$

Hence, the $L$-matrix of the Hubbard model is a representation of the graded Yang-Baxter algebra. This result is due to Olmedilla et al. [11]. To be self-contained we expose the $R$-matrix $\mathcal{R}(\lambda, \mu)$ along with some useful relations among its elements in appendix A. 
The graded tensor product in (43) respects comultiplication. Therefore the monodromy matrix

$$
\mathcal{T}_{L}(\lambda):=\mathcal{L}_{L}(\lambda) \ldots \mathcal{L}_{1}(\lambda)
$$

represents the graded Yang-Baxter algebra with the same $R$-matrix

$$
\mathcal{R}(\lambda, \mu)\left(\mathcal{T}_{L}(\lambda) \underset{\mathrm{S}}{\otimes} \mathcal{T}_{L}(\mu)\right)=\left(\mathcal{T}_{L}(\mu) \underset{\mathrm{S}}{\otimes} \mathcal{T}_{L}(\lambda)\right) \mathcal{R}(\lambda, \mu)
$$

We will demonstrate below that $\mathcal{T}_{L}(\lambda)$ generates the Hubbard Hamiltonian. For the matrix elements of $\mathcal{T}_{L}(\lambda)$ we introduce the following notation

$$
\mathcal{T}_{L}(\lambda)=\left(\begin{array}{cccc}
D_{11} & C_{11} & C_{12} & D_{12} \\
B_{11} & A_{11} & A_{12} & B_{12} \\
B_{21} & A_{21} & A_{22} & B_{22} \\
D_{21} & C_{21} & C_{22} & D_{22}
\end{array}\right),
$$

dividing it into four $2 \times 2$ submatrices $A(\lambda), B(\lambda), C(\lambda), D(\lambda)$. As we shall see in sections 5 and 6 , this block notation reflects the properties of the monodromy matrix under the two $\mathrm{su}(2)$ transformations connected with the Hubbard model and under combined particle-hole and gauge transformations.

If $\alpha, \beta, \gamma, \delta$ satisfy (42) and commute among each other and with the diagonal elements of $\mathcal{T}_{L}(\lambda)$, then (41), (44) and (46) imply that

$$
\left[\operatorname{tr}\left(\Gamma \mathcal{T}_{L}(\lambda)\right), \operatorname{tr}\left(\Gamma \mathcal{T}_{L}(\mu)\right)\right]=0
$$

Thus $\operatorname{tr}\left(\Gamma \mathcal{T}_{L}(\lambda)\right)$ generates a family of mutually commuting operators. Different choices of $\alpha, \beta, \gamma, \delta$ correspond to different boundary conditions. Since we did not restrict $\alpha$, $\beta, \gamma, \delta$ to be complex numbers, a dynamical twist is possible. In fact, because of the non-local nature of the Jordan-Wigner transformation, the periodic spin model turns into the Hubbard model with dynamically twisted boundary conditions. This point was recently discussed in detail by Yue and Deguchi [23]. To express the transfer matrix $t(\lambda)$ of the spin model introduced in section 2 in terms of fermi operators we note that $\Gamma=V_{L}^{-1}$ satisfies (42). Using (22) and (34) we conclude that

$$
t(\lambda)=\operatorname{tr}\left(V_{L}^{-1} \mathcal{T}_{L}(\lambda)\right)
$$


As we will see in the following, the Hubbard model under periodic boundary conditions is obtained with the choice $\Gamma=\sigma^{z} \otimes \sigma^{z}$, which leads to

$$
\operatorname{str}\left(\mathcal{T}_{L}(\lambda)\right):=\operatorname{tr}\left(\left(\sigma^{z} \otimes \sigma^{z}\right) \mathcal{T}_{L}(\lambda)\right)=\operatorname{tr}(D)-\operatorname{tr}(A)=\operatorname{str}\left(V_{L} T_{L}(\lambda)\right)
$$

This expression is called the graded trace or super trace of the monodromy matrix. Its zeroth order term of the expansion in the spectral parameter is

$$
\operatorname{str}\left(\mathcal{T}_{L}(0)\right)=\operatorname{str}\left(\mathcal{T}_{L \uparrow}(0)\right) \operatorname{str}\left(\mathcal{T}_{L \downarrow}(0)\right)=e^{-\mathrm{i} \pi u_{L} / 2} \hat{U}_{\uparrow} e^{-\mathrm{i} \pi d_{L} / 2} \hat{U}_{\downarrow}=e^{-\mathrm{i} \pi \hat{N} / 2} \hat{U}
$$

where $\hat{N}=u_{L}+d_{L}$ is the particle number operator, and $\hat{U}$ is the shift operator for electrons. (51) follows from the corresponding result for free fermions which we derive in appendix C. $\hat{U}$ is the product $\hat{U}=\hat{U}_{\uparrow} \hat{U}_{\downarrow}$ of shift operators for up and down spin electrons. We introduce these operators in appendix B, where we give a detailed account of shift and momentum operators for fermions on the lattice. $\hat{U}$ is connected to the total momentum $\Pi$ by $\hat{U}=e^{i \Pi}$. $\Pi$ assumes its familiar form,

$$
\Pi=\phi \sum_{k=1}^{L-1} k \tilde{c}_{k \tau}^{+} \tilde{c}_{k \tau}
$$

when expressed in terms of Fourier transformed fermi operators,

$$
\tilde{c}_{k \tau}=\frac{1}{\sqrt{L}} \sum_{l=1}^{L} e^{\mathrm{i} \phi k l} c_{l \tau} .
$$

For brevity we wrote $\phi:=2 \pi / L$ here. Eq. (52) has to be read with care, since $\Pi$ is merely defined modulo $2 \pi$. Hence we interpret (52) as defining equation of an equivalence class of operators differing from each other only by certain "phase operators". A restriction $\hat{\Pi}$ of $\Pi$ to the fundmental domain of the logarithm is constructed in appendix B. $\hat{\Pi}$ is a polynomial in $\hat{U}$.

The momentum operator $\hat{\Pi}$ preserves the particle number. Thus

$$
\ln \left(\operatorname{str}\left(\mathcal{T}_{L}(0)\right)\right)=-\mathrm{i} \pi \hat{N} / 2+\mathrm{i} \hat{\Pi}
$$

We will see moreover in section 6 that $\operatorname{str}\left(\mathcal{T}_{L}(\lambda)\right)$ commutes with the particle number operator and may therefore conclude that

$$
\left[\hat{\Pi}, \ln \left(\operatorname{str}\left(\mathcal{T}_{L}(\lambda)\right)\right)\right]=0
$$


This equation implies that $\tau(\lambda):=\ln \left(\operatorname{str}\left(\mathcal{T}_{L}(\lambda)\right)\right)$ is a generating function of translational invariant commuting operators. According to the arguments of Lüscher [24] these operators are local. They are most easily calculated in the language of the spin model, since the building blocks of the monodromy matrix are permutation operators of spins, $P_{j k \tau}=\frac{1}{2}\left(1+\sigma_{j \tau}^{\alpha} \sigma_{k \tau}^{\alpha}\right)$.

To give an example, we present the derivation of the Hamiltonian. Recall from section 2 that $\tilde{U}=t(0)$ is the shift operator for spins. If we reintroduce for a moment an auxiliary space index $a$, we obtain

$$
\dot{T}_{a L}(0)=P_{a 1} \tilde{U} \dot{L}_{L L-1}(0) P_{L L-1}+\sum_{j=1}^{L-1} \dot{L}_{j+1 j}(0) P_{j+1 j} P_{a 1} \tilde{U}
$$

The product $\dot{L}_{j k}(0) P_{j k}$ was given in section 2, eq. (14). Using the Jordan-Wigner transformation, (27), (28), it is expressed in terms of fermi operators as

$$
\dot{L}_{j+1 j}(0) P_{j+1 j}=c_{j \tau}^{+} c_{j+1 \tau}+c_{j+1 \tau}^{+} c_{j \tau}+U\left(n_{j \uparrow}-\frac{1}{2}\right)\left(n_{j \downarrow}-\frac{1}{2}\right)
$$

(50) and (51) imply $\operatorname{str}\left(V_{a L} P_{a 1} \tilde{U}\right)=e^{-\mathrm{i} \pi \hat{N} / 2} \hat{U}$. Since moreover $\left[V_{a L}, \dot{L}_{j+1 j}(0) P_{j+1 j}\right]=0$, we obtain $\operatorname{str}\left(\dot{\mathcal{T}}_{a L}(0)\right)=e^{-\mathrm{i} \pi \hat{N} / 2} \hat{U} \hat{H}$.

$\hat{H}$ is the Hubbard Hamiltonian

$$
\hat{H}=\sum_{j=1}^{L}\left(c_{j \tau}^{+} c_{j+1 \tau}+c_{j+1 \tau}^{+} c_{j \tau}+U\left(n_{j \uparrow}-\frac{1}{2}\right)\left(n_{j \downarrow}-\frac{1}{2}\right)\right)
$$

under periodic boundary conditions $\left(c_{L+1 \tau}:=c_{1 \tau}\right)$. Due to our choice of Jordan-Wigner transformation (27), (28) we obtained the Hamiltonian for holes here. The sign of the hopping term can of course be changed by a particle-hole transformation. Higher conserved quantities may be calculated in the same way as the Hamiltonian. We get an expansion of the generating function $\tau(\lambda)$, whose first terms are

$$
\tau(\lambda)=-\mathrm{i} \pi \hat{N} / 2+\mathrm{i} \hat{\Pi}+\lambda \hat{H}+\mathcal{O}\left(\lambda^{2}\right)
$$

The $\mathcal{O}\left(\lambda^{2}\right)$ term was derived by Shastry [9]. The zeroth order terms in (59) were not known before. They are however indispensable for the derivation of the dispersion relations of elementary excitations from the eigenvalues of $\operatorname{str}\left(\mathcal{T}_{L}(\lambda)\right)$ [16], 23]. It will be interesting to investigate, if we can get both branches of quasiparticle dispersion relations [3, 4] from these eigenvalues. 


\section{Discrete transformations}

A characteristic feature of the Hubbard Hamiltonian on a chain consisting of an even number of sites is its invariance under the transformation

$$
c_{j \uparrow} \rightarrow c_{j \uparrow} \quad, \quad c_{j \downarrow} \rightarrow(-1)^{j} c_{j \downarrow}^{+} \quad, \quad U \rightarrow-U
$$

Since the generators of rotations of the spins are not invariant under (60), there exists a second $\mathrm{su}(2)$ Lie algebra commuting with the Hamiltonian.

We will show now that not only the Hamiltonian, but the whole transfer matrix $\operatorname{str}\left(\mathcal{T}_{L}(\lambda)\right)$ is invariant under (60). First note that $h(\lambda) \rightarrow-h(\lambda)$, and thus

$$
G_{h} \rightarrow G_{-h}=G_{h}^{-1}
$$

The matrix elements of the free fermion $L$-matrix (31) transform according to

$$
\mathcal{L}_{k \downarrow}(\lambda) \rightarrow e^{\mathrm{i} \pi k \sigma^{z} / 2} \sigma^{y} \mathcal{L}_{k \downarrow}(\lambda) \sigma^{y} e^{-\mathrm{i} \pi(k-1) \sigma^{z} / 2}
$$

The last two formulae imply

$$
\mathcal{L}_{k}(\lambda) \rightarrow\left(I_{2} \otimes e^{\mathrm{i} \pi k \sigma^{z} / 2}\right)\left(\sigma^{z} \otimes \sigma^{y}\right) \mathcal{L}_{k}(\lambda)\left(\sigma^{z} \otimes \sigma^{y}\right)\left(I_{2} \otimes e^{-\mathrm{i} \pi(k-1) \sigma^{z} / 2}\right),
$$

and thus by comultiplication,

$$
\mathcal{T}_{L}(\lambda) \rightarrow\left(I_{2} \otimes e^{\mathrm{i} \pi L \sigma^{z} / 2}\right)\left(\sigma^{z} \otimes \sigma^{y}\right) \mathcal{T}_{L}(\lambda)\left(\sigma^{z} \otimes \sigma^{y}\right)
$$

Finally $\operatorname{str}\left(\mathcal{T}_{L}(\lambda)\right)$ transforms according to

$$
\operatorname{str}\left(\mathcal{T}_{L}(\lambda)\right) \rightarrow-e^{-\mathrm{i} \pi L / 2}\left(D_{11}(\lambda)-e^{\mathrm{i} \pi L} A_{11}(\lambda)-A_{22}(\lambda)+e^{\mathrm{i} \pi L} D_{22}(\lambda)\right)
$$

and we can conclude invariance modulo sign of the graded trace for even $L$

$$
\operatorname{str}\left(\mathcal{T}_{L}(\lambda)\right) \rightarrow \pm \operatorname{str}\left(\mathcal{T}_{L}(\lambda)\right)
$$

Hence, all higher commuting operators generated by $\tau(\lambda)=\ln \left(\operatorname{str}\left(\mathcal{T}_{L}(\lambda)\right)\right)$ are invariant under the transformation (60).

We can of course revers the spins in (60). Then a slight modification in the transformation of the monodromy matrix occurs. The factors $\sigma^{z} \otimes \sigma^{y}$ in (64) have to be 
replaced by $\sigma^{y} \otimes I_{2}$, and the two factors of $I_{2} \otimes e^{\mathrm{i} \pi L \sigma^{z} / 2}$ have to be interchanged. The result (66) for the transfer matrix remains the same. Performing up spin and down spin transformations in succession the monodromy matrix transforms as

$$
\mathcal{T}_{L}(\lambda) \rightarrow\left(e^{\mathrm{i} \pi L \sigma^{z} / 2} \otimes e^{\mathrm{i} \pi L \sigma^{z} / 2}\right)\left(\sigma^{x} \otimes \sigma^{y}\right) \mathcal{T}_{L}(\lambda)\left(\sigma^{x} \otimes \sigma^{y}\right)
$$

In this case we find for the transfer matrix

$$
\operatorname{str}\left(\mathcal{T}_{L}(\lambda)\right) \rightarrow \operatorname{tr}\left(e^{-\mathrm{i} \pi L \sigma^{z}} D(\lambda)\right)-\operatorname{tr}(A(\lambda))
$$

Again invariance is only achieved for an even number of lattice sites.

\section{$6 \quad \mathrm{su}(2)$ symmetries}

A careful discussion of the two $\mathrm{su}(2)$ symmetries [17, 18, 19, 20] connected with the Hubbard Hamiltonian was crucial for the complete understanding of the coordinate Bethe Ansatz of the model. It was shown in [25] that the states obtained from coordinate Bethe Ansatz are incomplete. They are highest weight states of two su(2) Lie algebras [26, 27]. One is the $\mathrm{su}(2)$ Lie algebra of rotations, the other one is the $\eta$-pairing $\mathrm{su}(2)$ Lie algebra. The generators of the $\eta$-pairing $\mathrm{su}(2)$ are obtained from the generators

of rotations under the canonical transformation of the preceding section. They are connected with the creation of pairs of particles or holes in the system.

We show in the following how the generators of the two symmetries commute with the monodromy matrix. Our result will be useful for the classification of quasiparticles according to their symmetry within the algebraic approach. A discussion analogous to the discussion of the spin of spin waves by Faddeev and Takhtajan 28] is likely to be possible. There are four interactionless states which may serve as reference states for an algebraic Bethe Ansatz of the Hubbard model, the empty band, the completely filled band and the half-filled band with all spins up or all spins down. Depending on the choice of reference state four of the elements of the matrices $B(\lambda)$ and $C(\lambda)$ in (47) are creation operators, whereas the remaining four are annihilation operators. This fits with the fact that there are four different quasiparticles in the system. We think that 
their identification will eventually become possible by use of the symmetry properties presented below.

The $\mathrm{su}(2)$ generators of rotations are given by

$$
S^{+}:=-\sum_{j=1}^{L} c_{j \uparrow}^{+} c_{j \downarrow} \quad, \quad S^{-}:=-\sum_{j=1}^{L} c_{j \downarrow}^{+} c_{j \uparrow} \quad, \quad S^{z}:=\sum_{j=1}^{L}\left(n_{j \uparrow}-n_{j \downarrow}\right)
$$

Recall that we are using the language of holes here (cf. (58)). Under a particle hole transformation the operators $S^{+}, S^{-}, S^{z}$ turn into the operators $\zeta^{\dagger}, \zeta, \zeta_{z}$ used by Eßler et al. [4]. We will show now that the whole transfer matrix is rotational invariant. To this end let us introduce local generators of rotations

$$
S_{j}^{+}:=-c_{j \uparrow}^{+} c_{j \downarrow} \quad, \quad S_{j}^{-}:=-c_{j \downarrow}^{+} c_{j \uparrow} \quad, \quad S_{j}^{z}:=n_{j \uparrow}-n_{j \downarrow} .
$$

The matrices

$$
\Sigma^{+}:=\sigma^{+} \otimes \sigma^{-} \quad, \quad \Sigma^{-}:=\sigma^{-} \otimes \sigma^{+} \quad, \quad \Sigma^{z}:=\frac{1}{2}\left(\sigma^{z} \otimes I_{2}-I_{2} \otimes \sigma^{z}\right)
$$

clearly generate a representation of $\mathrm{su}(2)$. They are connected to the inner block $A(\lambda)$ of the monodromy matrix $\mathcal{T}_{L}(\lambda)$. Let

$$
\begin{aligned}
& \Sigma^{x}:=\Sigma^{+}+\Sigma^{-}, \quad \Sigma^{y}:=-\mathrm{i}\left(\Sigma^{+}-\Sigma^{-}\right), \\
& S_{j}^{x}:=S_{j}^{+}+S_{j}^{-}, \quad S_{j}^{y}:=-\mathrm{i}\left(S_{j}^{+}-S_{j}^{-}\right) .
\end{aligned}
$$

Then it is not difficult to see that

$$
\left[\mathcal{L}_{j}(\lambda), \Sigma^{\alpha}+S_{j}^{\alpha}\right]=0 \quad, \quad \alpha=x, y, z
$$

The verification of this equation may be done as follows. First show by direct calculation that $\left[\mathcal{L}_{j}(\lambda), \Sigma^{+}+S_{j}^{+}\right]=0 . \mathcal{L}_{j}(\lambda)$ has the same block structure as the monodromy matrix, (47). Under reversing all spins the blocks $A(\lambda), B(\lambda), C(\lambda), D(\lambda)$ transform as

$$
\left(\begin{array}{ll}
A & B \\
C & D
\end{array}\right) \rightarrow\left(\begin{array}{cc}
\sigma^{x} & 0 \\
0 & \sigma^{z}
\end{array}\right)\left(\begin{array}{cc}
A & B \\
C & D
\end{array}\right)\left(\begin{array}{cc}
\sigma^{x} & 0 \\
0 & \sigma^{z}
\end{array}\right)
$$

and we may conclude that $\left[\mathcal{L}_{j}(\lambda), \Sigma^{-}+S_{j}^{-}\right]=0$. The vanishing of the last commutator $\left[\mathcal{L}_{j}(\lambda), \Sigma^{z}+S_{j}^{z}\right]$ follows by means of the Jacobi identity. 
The local equation (74) extends to an identity for the monodromy matrix by induction,

$$
\left[\mathcal{T}_{L}(\lambda), \Sigma^{\alpha}+S^{\alpha}\right]=0
$$

$\alpha=x, y, z$, where $S^{x}$ and $S^{y}$ are defined as their local analogs. Taking the graded trace of this equation yields

$$
\left[\operatorname{str}\left(\mathcal{T}_{L}(\lambda)\right), S^{\alpha}\right]=0 \quad, \quad \alpha=x, y, z
$$

The transfer matrix and thus all higher commuting operators are rotational invariant.

The transformation properties of the monodromy matrix under the discrete transformation (60) introduced in the preceding section induce a second $\mathrm{su}(2)$ invariance. Applying (60) to the su(2) generators of rotations, (69), we find

$$
\begin{aligned}
& S^{+} \rightarrow \eta^{+}=\sum_{j=1}^{L}(-1)^{j+1} c_{j \uparrow}^{+} c_{j \downarrow}^{+}, \\
& S^{-} \rightarrow \eta^{-}=\sum_{j=1}^{L}(-1)^{j+1} c_{j \downarrow} c_{j \uparrow}, \\
& S^{z} \rightarrow \eta^{z}=\sum_{j=1}^{L}\left(n_{j \uparrow}+n_{j \downarrow}-1\right)=\hat{N}-L .
\end{aligned}
$$

This is the $\eta$-pairing symmetry. Because of (80), it may be interpreted as non-abelian extension of the gauge symmetry. The commutators of the generators of $\eta$-pairing with the monodromy matrix follow from (60) and (76). Let

$$
\tilde{\Sigma}^{+}:=\sigma^{+} \otimes \sigma^{+} \quad, \quad \tilde{\Sigma}^{-}:=\sigma^{-} \otimes \sigma^{-} \quad, \quad \tilde{\Sigma}^{z}:=\frac{1}{2}\left(\sigma^{z} \otimes I_{2}+I_{2} \otimes \sigma^{z}\right) .
$$

These matrices generate a representation of $\mathrm{su}(2)$ connected to the block $D(\lambda)$ of the monodromy matrix. Like in case of rotations we define

$$
\begin{aligned}
\tilde{\Sigma}^{x}:=\tilde{\Sigma}^{+}+\tilde{\Sigma}^{-}, \quad \tilde{\Sigma}^{y}:=-\mathrm{i}\left(\tilde{\Sigma}^{+}-\tilde{\Sigma}^{-}\right), \\
\eta^{x}:=\eta^{+}+\eta^{-}, \quad \eta^{y}:=-\mathrm{i}\left(\eta^{+}-\eta^{-}\right) .
\end{aligned}
$$

Using these definitions we obtain for $L$ even

$$
\left[\mathcal{T}_{L}(\lambda), \tilde{\Sigma}^{\alpha}+\eta^{\alpha}\right]=0 \quad, \quad \alpha=x, y, z \quad
$$


and thus

$$
\left[\operatorname{str}\left(\mathcal{T}_{L}(\lambda)\right), \eta^{\alpha}\right]=0
$$

Note that there is no local analog like (74) of equation (85). The $\eta$-pairing symmetry is sensitive to a change of boundary conditions and is in this sense a non-local symmetry.

Equation (84) may be verified in the following way. Observe that

$$
\Sigma^{ \pm}\left(\sigma^{z} \otimes \sigma^{y}\right)=\left(\sigma^{z} \otimes \sigma^{y}\right) \tilde{\Sigma}^{ \pm}
$$

and

$$
\Sigma^{ \pm}\left(I_{2} \otimes e^{\mathrm{i} \pi L \sigma^{z} / 2}\right)=\left(I_{2} \otimes e^{-\mathrm{i} \pi L \sigma^{z} / 2}\right) \Sigma^{ \pm} .
$$

Apply the transformation (60) to equation (76), and use (86), (87). Then

$$
\left[\mathcal{T}_{L}(\lambda), \eta^{ \pm}\right]+\mathcal{T}_{L}(\lambda) \tilde{\Sigma}^{ \pm}-\left(I_{2} \otimes e^{\mathrm{i} \pi L \sigma^{z}}\right) \tilde{\Sigma}^{ \pm} \mathcal{T}_{L}(\lambda)=0
$$

Equation (87) remains true, if $\Sigma^{ \pm}$is replaced by $\tilde{\Sigma}^{ \pm}$. Using this fact, (88) implies

$$
\left[\mathcal{T}_{L}(\lambda), \tilde{\Sigma}^{z}+\eta^{z}\right]=0
$$

whereby

$$
\left[\operatorname{str}\left(\mathcal{T}_{L}(\lambda)\right), \hat{N}\right]=0
$$

for every $L$, which means that all higher conserved quantities are gauge invariant. This fact has been used in the derivation of (55). From (88) and (89) we infer the validity of (84) for even $L$.

Here is a simple example for the usefulness of the above formulae. (59), (80), (85) imply immediately that $\hat{\Pi} \eta^{+}=\eta^{+}(\hat{\Pi}+\pi)$, which means that $\eta^{+}$changes the momentum of eigenstates by $\pi[18]$.

\section{Conclusions}

We hope we could convince the reader, that the graded Yang-Baxter algebra (46) is a useful tool for further investigations of the one-dimensional Hubbard model. Our account of basic features of the monodromy matrix should be read in conjunction with the recent 
preprint of Ramos and Martins [16], who were able to diagonalize $\operatorname{str}\left(\mathcal{T}_{L}(\lambda)\right)$ by purely algebraic means. Combining both works it should be not too difficult to redrive algebraically all results obtained so far by means of the coordinate Bethe Ansatz. Moreover, there is hope to proceed in the calculation of correlation functions and thermodynamical properties.

There have been speculations [3] that there might be a different Yang-Baxter algebra embedding of the Hubbard Hamiltonian. Of course we can not rule out this possibility. Some arguments in favour of it, however, are disproved by now. In a recent article 14 we were able to show how a rational substructure of the $R$-matrix naturally arises in the thermodynamic limit. The corresponding submatrix of the monodromy matrix generates the $\mathrm{Y}(\mathrm{su}(2))$ representation discovered by Uglov and Korepin [13]. This nicely fits with the fact that the $S$-matrix of quasiparticle scattering is of rational form. In the present article we showed that the monodromy matrix has an appropriate algebraic structure. In particular, its graded trace is fully $\mathrm{su}(2) \oplus \mathrm{su}(2)$ invariant and invariant under translations. The analytic properties of $R$-matrix and monodromy matrix are less usual. We do not yet have a geometrical idea of the spectral curve. Still we succeeded in showing the existence of a meromorphic parametrization of the transfer matrix.

Acknowledgments. This work has been supported by the Japan Society for the Promotion of Science and the Ministry of Science, Culture and Education of Japan. We are grateful to Professor Miki Wadati for continuous encouragement and comments. We would like to thank H. Fehske, V. E. Korepin and M. Shiroishi for fruitful discussions. S. M. is also grateful to Professor Naoto Nagaosa for his encouragement. 


\section{Appendix A The R-matrix}

The $R$-matrix generating the graded Yang-Baxter algebra of the Hubbard model was first derived by Olmedilla et al. [11]. It follows from the equations (7), (23), (24) and (44) and is of the following structure,

$$
\begin{aligned}
& \mathcal{R}(\lambda, \mu)=\left(\begin{array}{cccccccccccccccc}
\rho_{1} & 0 & 0 & 0 & 0 & 0 & 0 & 0 & 0 & 0 & 0 & 0 & 0 & 0 & 0 & 0 \\
0 & \rho_{2} & 0 & 0 & \mathrm{i} \rho_{9} & 0 & 0 & 0 & 0 & 0 & 0 & 0 & 0 & 0 & 0 & 0 \\
0 & 0 & \rho_{2} & 0 & 0 & 0 & 0 & 0 & \mathrm{i} \rho_{9} & 0 & 0 & 0 & 0 & 0 & 0 & 0 \\
0 & 0 & 0 & \rho_{3} & 0 & 0 & -\mathrm{i} \rho_{6} & 0 & 0 & \mathrm{i} \rho_{6} & 0 & 0 & \rho_{8} & 0 & 0 & 0 \\
0 & -\mathrm{i} \rho_{10} & 0 & 0 & \rho_{2} & 0 & 0 & 0 & 0 & 0 & 0 & 0 & 0 & 0 & 0 & 0 \\
0 & 0 & 0 & 0 & 0 & \rho_{4} & 0 & 0 & 0 & 0 & 0 & 0 & 0 & 0 & 0 & 0 \\
0 & 0 & 0 & \mathrm{i} \rho_{6} & 0 & 0 & \rho_{5} & 0 & 0 & \rho_{7} & 0 & 0 & -\mathrm{i} \rho_{6} & 0 & 0 & 0 \\
0 & 0 & 0 & 0 & 0 & 0 & 0 & \rho_{2} & 0 & 0 & 0 & 0 & 0 & -\mathrm{i} \rho_{10} & 0 & 0 \\
0 & 0 & -\mathrm{i} \rho_{10} & 0 & 0 & 0 & 0 & 0 & \rho_{2} & 0 & 0 & 0 & 0 & 0 & 0 & 0 \\
0 & 0 & 0 & -\mathrm{i} \rho_{6} & 0 & 0 & \rho_{7} & 0 & 0 & \rho_{5} & 0 & 0 & \mathrm{i} \rho_{6} & 0 & 0 & 0 \\
0 & 0 & 0 & 0 & 0 & 0 & 0 & 0 & 0 & 0 & \rho_{4} & 0 & 0 & 0 & 0 & 0 \\
0 & 0 & 0 & 0 & 0 & 0 & 0 & 0 & 0 & 0 & 0 & \rho_{2} & 0 & 0 & -\mathrm{i} \rho_{10} & 0 \\
0 & 0 & 0 & \rho_{8} & 0 & 0 & \mathrm{i} \rho_{6} & 0 & 0 & -\mathrm{i} \rho_{6} & 0 & 0 & \rho_{3} & 0 & 0 & 0 \\
0 & 0 & 0 & 0 & 0 & 0 & 0 & \mathrm{i} \rho_{9} & 0 & 0 & 0 & 0 & 0 & \rho_{2} & 0 & 0 \\
0 & 0 & 0 & 0 & 0 & 0 & 0 & 0 & 0 & 0 & 0 & \mathrm{i} \rho_{9} & 0 & 0 & \rho_{2} & 0 \\
0 & 0 & 0 & 0 & 0 & 0 & 0 & 0 & 0 & 0 & 0 & 0 & 0 & 0 & 0 & \rho_{1}
\end{array}\right)
\end{aligned}
$$

The ten Boltzmann weights $\rho_{j}=\rho_{j}(\lambda, \mu)$ are

$$
\begin{aligned}
& \rho_{1}=\cos (\lambda) \cos (\mu) e^{h-l}+\sin (\lambda) \sin (\mu) e^{l-h}, \\
& \rho_{2}=1, \\
& \rho_{3}=\frac{\cos (\lambda) \cos (\mu) e^{h-l}-\sin (\lambda) \sin (\mu) e^{l-h}}{\cos ^{2}(\lambda)-\sin ^{2}(\mu)}, \\
& \rho_{4}=\cos (\lambda) \cos (\mu) e^{l-h}+\sin (\lambda) \sin (\mu) e^{h-l},
\end{aligned}
$$




$$
\begin{aligned}
\rho_{5} & =\frac{\cos (\lambda) \cos (\mu) e^{l-h}-\sin (\lambda) \sin (\mu) e^{h-l}}{\cos ^{2}(\lambda)-\sin ^{2}(\mu)}, \\
\rho_{6} & =\frac{2 \operatorname{sh}(2(h-l))}{U\left(\cos ^{2}(\lambda)-\sin ^{2}(\mu)\right)}, \\
\rho_{7} & =\rho_{4}-\rho_{5} \quad, \\
\rho_{8} & =\rho_{1}-\rho_{3} \quad, \\
\rho_{9} & =\sin (\lambda) \cos (\mu) e^{l-h}-\cos (\lambda) \sin (\mu) e^{h-l}, \\
\rho_{10} & =\sin (\lambda) \cos (\mu) e^{h-l}-\cos (\lambda) \sin (\mu) e^{l-h}
\end{aligned}
$$

The parameters $\lambda, \mu, h$ and $l$ are connected by equation (9). Note that our definition of $h$ and $l$ differs from that in ref. [11] and that we performed a shift of $\frac{\pi}{4}$ in the arguments of the functions $\alpha(\lambda)$ and $\gamma(\lambda)$ occurring there.

There are the following quadratic relations between the Boltzmann weights, [11],

$$
\begin{gathered}
\rho_{1} \rho_{4}+\rho_{9} \rho_{10}=1 \\
\rho_{1} \rho_{5}+\rho_{3} \rho_{4}=2 \\
\rho_{3} \rho_{5}-\rho_{6}^{2}=1
\end{gathered},
$$

Further identities useful for practical calculations can be found in the recent article 14.

\section{Appendix B Momentum operator for fermions on a lattice}

Below we present a detailed discussion of the momentum operator on a lattice.

\section{The shift operator from permutations}

In this subsection we mimic the construction of the shift operator for spin chains. Start with spinless fermions, $c_{1}, \ldots, c_{L}$ on a one dimensional lattice of $L$ sites. Let

$$
K_{i j}:=1-\left(c_{i}^{+}-c_{j}^{+}\right)\left(c_{i}-c_{j}\right)
$$


It is not difficult to see that $K_{i j}$ permutes fermions. There are the obvious identities

$$
K_{i j}=K_{i j}^{+} \quad, \quad K_{i j}=K_{j i} \quad, \quad K_{j j}=1
$$

Use of the fundamental anti commutators for the fermions yields

$$
K_{i j} c_{i}=c_{i}+\left(c_{i}^{+}-c_{j}^{+}\right) c_{j}\left(c_{i}-c_{j}\right)=c_{i}+\left(-1+c_{j} c_{j}^{+}-c_{j} c_{i}^{+}\right)\left(c_{i}-c_{j}\right)=c_{j} K_{i j},
$$

and (B.2) implies

$$
K_{i j} c_{j}=c_{i} K_{i j} \quad, \quad K_{i j} c_{i}^{+}=c_{j}^{+} K_{i j} \quad, \quad K_{i j} c_{j}^{+}=c_{i}^{+} K_{i j}
$$

Furthermore, by the last four equations, we obtain

$$
K_{i j} K_{j k}=K_{i k} K_{i j}=K_{j k} K_{i k} \quad, \quad i \neq j \neq k
$$

and a short calculation similar to the one in eq. (B.3) leads to

$$
K_{i j} K_{i j}=1
$$

Hence, the operators $K_{i j}$ are identified as permutation operators.

With the aid of permutations of fermions it is of course possible to realize a global shift [17]. Let

$$
\hat{U}:=K_{12} K_{23} \ldots K_{L-1 L}
$$

Then eq. (B.3) implies

$$
\hat{U} c_{j}=\left\{\begin{array}{ll}
c_{j+1} \hat{U} \\
c_{1} \hat{U}
\end{array} \quad, \quad \text { if } \begin{array}{l}
j=1, \ldots, L-1 \\
j=L .
\end{array}\right.
$$

This means that $\hat{U}$ is acting as right shift operator on the elementary fermi operators of a periodic chain of $L$ sites. Now (B.7) implies

$$
\hat{U}^{+}=K_{L-1 L} \ldots K_{12}
$$

and thus by eq. (B.6), $\hat{U} \hat{U}^{+}=\hat{U}^{+} \hat{U}=1$. $\hat{U}$ is invertible, and $\hat{U}^{-1}=\hat{U}^{+}$, i.e. $\hat{U}$ is unitary. $\hat{U}^{-1}$ is the left shift operator. With the aid of the shift operator it is possible to define the momentum as its formal infinitesimal generator,

$$
e^{\mathrm{i} \Pi}:=\hat{U}
$$


This is the common construction in case of the spin chains. Note however, that (B.10) defines $\Pi$ only modulo $2 \pi$.

To realize the shift operator for electrons, attach a spin label to all operators above, and observe that $\left[\hat{U}_{\uparrow}, c_{j \downarrow}\right]=\left[\hat{U}_{\downarrow}, c_{j \uparrow}\right]=0$. Then $\hat{U}:=\hat{U}_{\uparrow} \hat{U}_{\downarrow}$ is the shift operator for electrons.

\section{Diagonalization of the momentum operator - an exercise}

For simplicity, return to the spinless case. Because of the basic property (B.8), it follows that $\left[\hat{U}^{L}, c_{j}\right]=\left[\hat{U}^{L}, c_{j}^{+}\right]=0, j=1, \ldots, L$, i.e. $\hat{U}^{L}$ is a scalar operator. Since $\hat{U}|0\rangle=|0\rangle$, we obtain $\hat{U}^{L}=1$. Shifting all fermions once around the lattice does not change the state of the system. This simple fact has strong implications.

Let $\alpha \in \mathbf{C}$. Then

$$
(1-\alpha \hat{U}) \sum_{n=0}^{L-1} \alpha^{n} \hat{U}^{n}=1-\alpha^{L} .
$$

This means that the resolvent of $\hat{U}$ is a finite sum. With $\lambda:=1 / \alpha$ we find,

$$
(\lambda-\hat{U})^{-1}=\frac{1}{\lambda\left(\lambda^{L}-1\right)} \sum_{n=0}^{L-1} \lambda^{L-n} \hat{U}^{n}
$$

The sum on the right hand side of the latter equation is regular in $\lambda$ and of order $\lambda$. The spectrum of $\hat{U}$ is therefore given by the equation

$$
\lambda^{L}=1 \quad, \quad \Leftrightarrow \quad, \quad \lambda_{k}=e^{\mathrm{i} 2 \pi k / L} \quad, \quad k=0, \ldots, L-1
$$

and is of course highly degenerate.

Let $P_{0}, \ldots, P_{L-1}$ be the projections on the eigenspaces of $\hat{U}$ corresponding to the eigenvalues $\lambda_{0}, \ldots, \lambda_{L-1}$. The $P_{k}$ are orthogonal, since the eigenstates of a unitary operator to different eigenvalues are. $P_{k} P_{l}=\delta_{k l} P_{k}$, and furthermore, $\sum_{k=0}^{L-1} P_{k}=1$. Hence, the spectral decomposition of the resolvent (B.12) is

$$
(\lambda-\hat{U})^{-1}=\sum_{k=0}^{L-1} \frac{P_{k}}{\lambda-\lambda_{k}} .
$$


Conversely, the $P_{k}$ are determined by the spectral decomposition via $P_{k}=\operatorname{res}_{\lambda=\lambda_{k}}((\lambda-$ $\left.\hat{U})^{-1}\right)$. Since $\left.d_{\lambda}\left(\lambda\left(\lambda^{L}-1\right)\right)\right|_{\lambda=\lambda_{k}}=L$, we obtain

$$
P_{k}=\frac{1}{L} \sum_{n=0}^{L-1} e^{-\mathrm{i} \phi k n} \hat{U}^{n}
$$

where we used the abbreviation $\phi=2 \pi / L$. From this representation the momentum eigenstates arise quite naturally. The particle number operator $\hat{N}$ is of course translational invariant, $[\hat{N}, \hat{U}]=0$. Hence, the projections $P_{k}$ preserve the particle number, $\left[P_{k}, \hat{N}\right]=0$. We find

$$
P_{k} c_{j}^{+}|0\rangle=\frac{e^{\mathrm{i} \phi j k}}{L} \sum_{n=1}^{L} e^{-\mathrm{i} \phi k n} c_{n}^{+}|0\rangle
$$

Since this is true for all $j=1, \ldots, L$, we see that $\hat{U}$ is nondegenerate in the one particle sector of the Hilbert space. In this sector the subspace corresponding to the eigenvalue $\lambda_{k}$ is spanned by the right hand side of the above equation. Normalization yields the eigenvector $|k\rangle:=\tilde{c}_{k}^{+}|0\rangle$, where $\tilde{c}_{k}^{+}$is the creator of a one particle momentum eigenstate. It is defined in the usual way as the adjoint of the annihilator

$$
\tilde{c}_{k}:=\frac{1}{\sqrt{L}} \sum_{n=1}^{L} e^{\mathrm{i} \phi k n} c_{n} .
$$

$\tilde{c}_{k}$ and $\tilde{c}_{k}^{+}$are fermi operators. They are transformed back into site operators $c_{j}, c_{j}^{+}$by Fourier inversion of eq. (B.17). Therefore arbitrary operators defined in terms of $c_{j}, c_{j}^{+}$ may be expressed in terms of $\tilde{c}_{k}$ and $\tilde{c}_{k}^{+}$. The particle number operator, in particular, is form invariant under Fourier transformation,

$$
\hat{N}=\sum_{k=0}^{L-1} \tilde{c}_{k}^{+} \tilde{c}_{k}
$$

Since the $\tilde{c}_{k}$ are fermi operators, the states $\left|k_{1} \ldots k_{N}\right\rangle:=\tilde{c}_{k_{1}}^{+} \ldots \tilde{c}_{k_{N}}^{+}|0\rangle, k_{1}<\ldots<k_{N}$, are orthogonal. (B.18) implies $\hat{N}\left|k_{1} \ldots k_{N}\right\rangle=N\left|k_{1} \ldots k_{N}\right\rangle$. Counting these states we see that they span the $N$-particle sector of the lattice Hilbert space. Letting $N$ vary from $1, \ldots, L$ we get a basis of the full Hilbert space.

Now, from the definition $(\mathbb{B} .17)$ of $\tilde{c}_{k}$ it follows that

$$
\hat{U} \tilde{c}_{k}^{+}=e^{\mathrm{i} \phi k} \tilde{c}_{k}^{+} \hat{U}
$$


Hence we have obtained

$$
\hat{U}\left|k_{1} \ldots k_{N}\right\rangle=e^{\mathrm{i} \phi\left(k_{1}+\ldots+k_{N}\right)}\left|k_{1} \ldots k_{N}\right\rangle
$$

or for the momentum operator, respectively,

$$
\Pi\left|k_{1} \ldots k_{N}\right\rangle=\phi\left(k_{1}+\ldots+k_{N}\right)\left|k_{1} \ldots k_{N}\right\rangle
$$

This equation is of course defined only modulo $2 \pi$. On the other hand it is easily verified that the operator $\phi \sum_{k=1}^{L-1} k \tilde{c}_{k}^{+} \tilde{c}_{k}$ acts the same way on $\left|k_{1} \ldots k_{N}\right\rangle$. Since these states form a basis, we have achieved the diagonalization of the momentum operator,

$$
\Pi=\phi \sum_{k=1}^{L-1} k \tilde{c}_{k}^{+} \tilde{c}_{k}
$$

In this form $\Pi$ is usually found in the literature.

\section{Site representation of the momentum operator}

What happens, when we use the Fourier transform to translate back the above diagonal form of the momentum operator into the site representation? Inserting the definition (B.17) into eq. (B.22) yields

$$
\Pi=\frac{\phi}{L} \sum_{m, n=1}^{L} c_{m}^{+} c_{n} \sum_{k=1}^{L-1} k e^{-\mathrm{i} \phi(m-n) k}
$$

Now, for $\alpha \in \mathbf{C}$, let

$$
g(\alpha):=\sum_{k=0}^{L-1} \mathrm{i} e^{-\mathrm{i} \phi k \alpha}=\mathrm{i} \frac{1-e^{-\mathrm{i} \phi L \alpha}}{1-e^{-\mathrm{i} \phi \alpha}},
$$

where the term on the right hand side makes sense only for $\alpha \notin L \mathbf{Z}$. Eqs. (B.23) and (B.24) imply that

$$
\begin{aligned}
\Pi & =\frac{1}{L} \sum_{m, n=1}^{L} g^{\prime}(m-n) c_{m}^{+} c_{n} \\
& =\frac{1}{2} \phi(L-1) \hat{N}+\phi \sum_{\substack{m, n=1 \\
m \neq n}}^{L} \frac{c_{m}^{+} c_{n}}{e^{-\mathrm{i} \phi(m-n)}-1} .
\end{aligned}
$$

This formula is remarkable. Since $\Pi=-i \ln (\hat{U})$, we can read it as having taken the logarithm of the ordered product of transpositions which defines $\hat{U}$. $\Pi$ as given by 
$(\overline{B .25})$ is a non-local operator. It is interesting to notice that the second term on the right hand side of $(\mathbb{B . 2 5})$ is gauge equivalent to the $1 / \sin (\phi(m-n))$ hopping term of the long-range Hubbard model introduced by Gebhard and Ruckenstein [29. We have thus found a simple interpretation of this Hamiltonian and in turns a simple interpretation of the origin of the $1 / \sin ^{2}$ exchange of the Haldane-Shastry spin chain [30, 31].

\section{Momentum operator modulo $2 \pi$}

To obtain the appropriate definition of $\Pi \bmod 2 \pi$, observe from the preceding subsection that

$$
\phi k=\frac{1}{L} \sum_{m=1}^{L} g^{\prime}(m) e^{\mathrm{i} \phi k m}=\phi \sum_{m=1}^{L-1}\left(\frac{1}{2}+\frac{e^{\mathrm{i} \phi k m}}{e^{-\mathrm{i} \phi m}-1}\right)
$$

for $k=0, \ldots, L-1$. With view of the right hand side of this equation the function $\phi k$ is periodically continued to a saw tooth function on the integers. Since $\Pi / \phi$ assumes only integer eigenvalues, the definition

$$
\hat{\Pi}:=\phi \sum_{m=1}^{L-1}\left(\frac{1}{2}+\frac{\hat{U}^{m}}{e^{-\mathrm{i} \phi m}-1}\right)
$$

yields the required restriction of $\Pi$ modulo $2 \pi$. In other words, (B.19) and (B.26) imply that $e^{\mathrm{i} \Pi}=e^{\mathrm{i} \hat{\Pi}}$. $\hat{\Pi}$ obviously commutes with the Hubbard Hamiltonian, whereas $\Pi$ does not.

\section{Appendix C Free fermions}

In this appendix we show how to obtain the shift operator $\hat{U}$ for fermions from the graded trace of the free fermion monodromy matrix (33). For this purpose we have to treat XX-chain and free fermion model in parallel. Both models are related by a Jordan-Wigner transformation. For brevity let us consider the up-spin case (27), and let us suppress the spin index here. The XX-chain monodromy matrix is

$$
T_{L}(\lambda)=l_{L}(\lambda) \ldots l_{1}(\lambda)
$$

It satisfies the Yang-Baxter algebra (5),

$$
\check{r}(\lambda-\mu)\left(T_{L}(\lambda) \otimes T_{L}(\mu)\right)=\left(T_{L}(\mu) \otimes T_{L}(\lambda)\right) \check{r}(\lambda-\mu)
$$


Applying the Jordan-Wigner transformation (27) to the XX-chain monodromy matrix, we obtain

$$
T_{L}(\lambda)=e^{-\mathrm{i} \pi u_{L} \sigma^{z} / 2} \mathcal{T}_{L}(\lambda)
$$

where $\mathcal{T}_{L}(\lambda)$ is the free fermion monodromy matrix (33) with $\tau=\uparrow$, which satisfies the graded analog of (C.2),

$$
\check{r}_{g}(\lambda-\mu)\left(\mathcal{T}_{L}(\lambda) \underset{\mathrm{S}}{\otimes} \mathcal{T}_{L}(\mu)\right)=\left(\mathcal{T}_{L}(\mu) \underset{\mathrm{S}}{\otimes} \mathcal{T}_{L}(\lambda)\right) \check{r}_{g}(\lambda-\mu)
$$

The matrix $\check{r}_{g}(\lambda)$ is defined as $\check{r}_{g}(\lambda):=W^{-1} \check{r}(\lambda) W$ with $W$ according to (36). (C.4) implies that

$$
\left[\operatorname{str}\left(\mathcal{T}_{L}(\lambda)\right), \operatorname{str}\left(\mathcal{T}_{L}(\mu)\right)\right]=0
$$

We want to calculate the action of $\operatorname{str}\left(\mathcal{T}_{L}(0)\right)$ on fermi operators. To this end let

$$
P_{0 j}:=\frac{1}{2}\left(1+\sigma^{\alpha} \sigma_{j}^{\alpha}\right) \quad, \quad P_{j k}:=\frac{1}{2}\left(1+\sigma_{j}^{\alpha} \sigma_{k}^{\alpha}\right)
$$

Then (C.1) implies that

$$
T_{L}(0)=P_{01} P_{12} \ldots P_{L-1 L}=P_{01} \tilde{U}
$$

We use the Jordan-Wigner transformation (27) to express $P_{j j+1}$ in terms of fermi operators. Then

$$
P_{j j+1}=K_{j j+1}+2 n_{j} n_{j+1}
$$

with the spinless densities $n_{j}=c_{j}^{+} c_{j}$ and the permutation operators $K_{j j+1}$ according to (B.1). Let $e_{ \pm}:=e^{ \pm \mathrm{i} \pi u_{L} / 2}$. Then (C.3) implies that

$$
\operatorname{str}\left(\mathcal{T}_{L}(0)\right)=\left(e_{+} n_{1}+e_{-}\left(n_{1}-1\right)\right) \tilde{U}
$$

Using the Jordan-Wigner transformation (27) and the known action of the permutation operators $P_{j j+1}$ on Pauli matrices, we obtain

$$
\begin{aligned}
& \tilde{U} c_{k}=c_{k+1}\left(1-2 n_{1}\right) \tilde{U} \quad, \quad k=1, \ldots, L-1 \\
& \tilde{U} c_{L}=c_{1}\left(1-2 n_{1}\right) e_{+}^{2} \tilde{U}
\end{aligned}
$$


$e_{+}$and $e_{-}$are generators of gauge transformations,

$$
e_{+} c_{k}=-\mathrm{i} c_{k} e_{+} \quad, \quad e_{-} c_{k}=\mathrm{i} c_{k} e_{-}
$$

By use of the four previous formulae, we infer for the annihilators $c_{k}$,

$$
\operatorname{str}\left(\mathcal{T}_{L}(0)\right) c_{k}=\mathrm{i} c_{k+1} \operatorname{str}\left(\mathcal{T}_{L}(0)\right)
$$

where $k=1, \ldots, L\left(c_{L+1}=c_{1}\right)$. It is not difficult to see that $\operatorname{str}\left(\mathcal{T}_{L}(0)\right)$ is a unitary operator. Therefore the creators $c_{k}^{+}$satisfy

$$
\operatorname{str}\left(\mathcal{T}_{L}(0)\right) c_{k}^{+}=-\mathrm{i} c_{k+1}^{+} \operatorname{str}\left(\mathcal{T}_{L}(0)\right)
$$

$k=1, \ldots, L$. (C.12), (C.13) and (C.14) imply that

$$
e_{+} \operatorname{str}\left(\mathcal{T}_{L}(0)\right)=\alpha_{L} \hat{U}
$$

where $\alpha_{L}$ is a complex constant, and $\hat{U}$ is the shift operator (B.7) for spinless fermions. Since $K_{j j+1}|0\rangle=P_{j j+1}|0\rangle=|0\rangle$, we conclude from (C.9) that $\alpha_{L}=-1$ and eventually obtain

$$
\operatorname{str}\left(\mathcal{T}_{L}(0)\right)=-e_{-} \hat{U}
$$

The corresponding formula for down-spins is simply obtained by reversing the spins. Then (C.16) implies (51). It may be instructive for the reader to verify (C.16) directly for small $L$.

\section{References}

[1] E. H. Lieb and F. Y. Wu, Phys. Rev. Lett. 20, 1445 (1968).

[2] F. H. L. Eßler and V. E. Korepin, Exactly Solvable Models of Strongly Correlated Electrons, World Scientific, Singapore, (1994).

[3] F. H. L. Eßler and V. E. Korepin, Phys. Rev. Lett. 72, 908 (1994).

[4] F. H. L. Eßler and V. E. Korepin, Nucl. Phys. B 426, 505 (1994). 
[5] H. Frahm and V. E. Korepin, Phys. Rev. B 43, 5653 (1991).

[6] A. Klümper and R. Z. Bariev, Nucl. Phys. B 458, 623 (1996).

[7] B. S. Shastry, Phys. Rev. Lett. 56, 1529 (1986).

[8] B. S. Shastry, Phys. Rev. Lett. 56, 2453 (1986).

[9] B. S. Shastry, J. Stat. Phys. 50, 57 (1988).

[10] M. Wadati, E. Olmedilla, and Y. Akutsu, J. Phys. Soc. Jpn. 56, 1340 (1987).

[11] E. Olmedilla, M. Wadati, and Y. Akutsu, J. Phys. Soc. Jpn. 56, 2298 (1987).

[12] E. Olmedilla and M. Wadati, Phys. Rev. Lett. 60, 1595 (1988).

[13] D. B. Uglov and V. E. Korepin, Phys. Lett. A 190, 238 (1994).

[14] S. Murakami and F. Göhmann. Yangian Symmetry and Quantum Inverse Scattering Method for the One-Dimensional Hubbard Model. preprint, cond-mat/9609249, (1996).

[15] M. Shiroishi and M. Wadati, J. Phys. Soc. Jpn. 64, 57 (1995).

[16] P. B. Ramos and M. J. Martins. Algebraic Bethe Ansatz Approach for the OneDimensional Hubbard Model. preprint, hep-th/9605141, (1996).

[17] O. J. Heilmann and E. H. Lieb, Ann. N.Y. Acad. Sci. 172, 584 (1971).

[18] C. N. Yang, Phys. Rev. Lett. 63, 2144 (1989).

[19] C. N. Yang and S. C. Zhang, Mod. Phys. Lett. B 4, 40 (1990).

[20] M. Pernici, Europhys. Lett. 12, 75 (1990).

[21] R. J. Baxter, Exactly Solved Models in Statistical Mechanics, Academic Press, London, (1982).

[22] P. P. Kulish and E. K. Sklyanin, J. Soviet Math. 19, 1596 (1982). 
[23] R. Yue and T. Deguchi. Analytic Bethe Ansatz for 1-d Hubbard model and twisted coupled XY model. preprint, hep-th/9605141, (1996).

[24] M. Lüscher, Nucl. Phys. B 117, 475 (1976).

[25] F. H. L. Eßler, V. E. Korepin, and K. Schoutens, Nucl. Phys. B 372, 559 (1992).

[26] F. H. L. Eßler, V. E. Korepin, and K. Schoutens, Phys. Rev. Lett. 67, 3848 (1991).

[27] F. H. L. Eßler, V. E. Korepin, and K. Schoutens, Nucl. Phys. B 384, 431 (1992).

[28] L. A. Takhtajan and L. D. Faddeev, J. Soviet Math. 24, 241 (1984).

[29] F. Gebhard and A. E. Ruckenstein, Phys. Rev. Lett. 68, 244 (1992).

[30] F. D. M. Haldane, Phys. Rev. Lett. 60, 635 (1988).

[31] B. S. Shastry, Phys. Rev. Lett. 60, 639 (1988). 